\title{
Towards a performance theory of robust adaptive control
}

\author{
Ahmad Sanei and Mark French ${ }^{*, \dagger}$ \\ School of Electronics and Computer Science, University of Southampton, Southampton SO17 1BJ, U.K.
}

\begin{abstract}
SUMMARY
We consider standard robust adaptive control designs based on the dead-zone and projection modifications, and compare their performance w.r.t. a worst case transient cost functional penalizing the $\mathscr{L}^{\infty}$ norm of the output, control and control derivative. If a bound on the $\mathscr{L}^{\infty}$ norm of the disturbance is known, it is shown that the dead-zone controller outperforms the projection controller if the a priori information on the uncertainty level is sufficiently conservative. The second result shows that the projection controller is superior to the dead-zone controller when the a priori information on the disturbance level is sufficiently conservative. For conceptual clarity the results are presented on a non-linear scalar system with a single uncertain parameter and generalizations are briefly discussed. Copyright (C) 2004 John Wiley \& Sons, Ltd.
\end{abstract}

KEY WORDS: non-linear adaptive control; transient performance; dead-zone; projection

\section{INTRODUCTION}

It is well known that adaptive controllers are susceptible to phenomena such as parameter drift even when small input disturbances are present. To overcome such problems, a number of standard techniques are widely utilized, such as the dead-zones modification, the $\sigma$-modification or the projection modification [1-6].

Each of these techniques have advantages and drawbacks. For example, as is well known, dead-zone modifications generally require a priori knowledge of the disturbance level, and only achieve convergence of the output to some pre-specified neighbourhood of the origin (whilst keeping all signals bounded). In particular, if the disturbances vanish, then dead-zone controllers do not typically achieve convergence of the output to zero: the convergence remains to the pre-specified neighbourhood of the origin. On the other hand, it is also well known that projection modifications generally achieve boundedness of all signals, and furthermore have the desirable property that if no disturbances are present, then the output converges to zerohowever, an arbitrarily small $\mathscr{L}^{\infty}$ disturbance can completely destroy any convergence of the output. An extensive discussion of $\sigma$-modification can be found in Reference [7].

\footnotetext{
*Correspondence to: M. French, School of Electronics and Computer Science, University of Southampton, Southampton SO17 1BJ, U.K.

†E-mail: mcf@ecs.soton.ac.uk

Published online 29 March 2004

Copyright (C) 2004 John Wiley \& Sons, Ltd.

Received 14 March 2003

Revised 12 June 2003 Accepted 17 October 2003
} 
This illustrates that in the case of asymptotic performance, there are some well-known advantages and disadvantages of the various robust-adaptive schemes. However, there are many situations in which we cannot definitively state whether, e.g. a projection or dead-zone controller is superior even when only considering asymptotic performance. Furthermore, the known results, as with most results in adaptive control, are confined to non-singular performances, i.e. without any consideration of the control signal.

The goal of this paper is to compare dead-zone and projection based adaptive controllers with respect to a transient performance measure $\mathscr{P}$. Furthermore, the transient performance measure will be non-singular (i.e. penalize both the state $(x)$ and the input $(u)$ of the plant); specifically we will consider cost functionals which penalize the state $\left(\|x(\cdot)\|_{\mathscr{L}^{\infty}}\right)$, control $\left(\|u(\cdot)\|_{\mathscr{L}^{\infty}}\right)$ and control rate $\left(\|\dot{u}(\cdot)\|_{\mathscr{L}^{\infty}}\right.$.). In the case of a scalar non-linear plant, we will identify circumstances in which a dead-zone based adaptive controller is superior to the projection based adaptive controller with respect to $\mathscr{P}$, and vice versa. A scalar system has been chosen on which to develop the results to illustrate the trade-offs between the designs in the simplest manner: however note that there is a certain level of technical difficulty even with such simple plants.

In Section 5, we outline extensions to more general classes of systems, in particular to nonlinear integrator chains, and to minimum phase linear systems of relative degree one with positive high-frequency gain. The proofs of these more complex results are substantively more involved and can be found in References $[8,9]$.

This is a new direction in adaptive control theory-to date there are few results in adaptive control which consider transient performance costs which penalize control effort. The inherent trade-offs which lie in the choice between designs are central to any quantitative control theory, and it should be observed that such trade-offs can only be studied in a non-singular performance framework.

We conclude by indicating the directions for future work.

\section{STATEMENT OF THE PROBLEM AND MAIN RESULT}

\subsection{System and basic control design}

Consider the following class of SISO non-linear system:

$$
\Sigma\left(x_{0}, \theta, d(\cdot)\right): \dot{x}(t)=\theta \phi(x(t))+u(t)+d(t), \quad x(0)=x_{0}
$$

where $x(\cdot) u(\cdot), \theta \in \mathbb{R}$ are the state vector, the control input, and unknown constant parameter, respectively. $d(\cdot)$ belongs to a class of bounded disturbances $\mathscr{D} \subset \mathscr{L}^{\infty}[0, \infty)$ and $\phi(\cdot)$ is a known smooth real valued function which is assumed to satisfy some or all of the following conditions at various points in the paper:

$$
\begin{aligned}
& \text { (a) } x=0 \Leftrightarrow \phi(x)=0 \\
& \text { (b) }\left.\frac{\partial \phi(x)}{\partial x}\right|_{x=0}>0 \\
& \text { (c) } \inf _{x \in \mathbb{R}}\left|\frac{\phi(x)}{x}\right| \geqslant \beta>0
\end{aligned}
$$


These conditions specify that the system with no inputs $(u=d=0)$ has an unstable equilibrium at the origin, and that the non-linearity has at least linear growth.

It can be easily shown (see e.g. Reference [5]) that disturbance free $(\mathscr{D}=\{0\})$ systems of form (1) are stabilized by the following simple adaptive controller:

$$
\begin{aligned}
\Xi: u(t) & =-a x(t)-\hat{\theta}(t) \phi(x(t)) \\
\dot{\hat{\theta}}(t) & =\alpha x(t) \phi(x(t)), \quad \hat{\theta}(0)=0
\end{aligned}
$$

where $\hat{\theta}(\cdot)$ is an adaptive estimator of $\theta, \alpha>0$ is the adaptation gain, and $a>0$ is the control gain.

\subsection{Robust modifications to the control design}

It is well known that even a small $\mathscr{L}^{\infty}$ disturbance can cause the parameter estimate $\hat{\theta}(\cdot)$ to diverge, see e.g. References $[1,5]$. Such a phenomenon is typically called 'parameter drift'. To overcome this problem, two distinct approaches have been proposed: (i) using an appropriately rich reference input to achieve persistent excitation of the identifier and hence convergence of the parameter estimator, and (ii) modification of the adaptation law. In this section, we briefly explain the two common methods for modifying the adaptive law which form the basis of this paper: the dead-zone and the parameter projection modifications.

2.2.1. Dead-zone modification. The idea of dead-zone [6] is to modify the parameter estimator so that the adaptive mechanism is 'switched off' when system trajectory $x(\cdot)$ lies inside a region $\Omega_{0}$ where the disturbance has a destabilising effect on the dynamics. A priori knowledge of the size of the disturbance is typically used to define the size of the dead-zone. Let $d_{\max }$ be the a priori known upper bound of the disturbance level, i.e. $d_{\max } \geqslant\|d(\cdot)\|_{\mathscr{L}^{\infty}}$ for all $d(\cdot) \in \mathscr{D}$. For scalar systems (1), the dead-zone region $\Omega_{0}\left(d_{\max }\right)$ can be simply defined by $\Omega_{0}\left(d_{\max }\right)=\left[-\eta_{0}, \eta_{0}\right]$, where $\eta_{0}=\varrho\left(d_{\max }\right)$ and $\varrho: \mathbb{R}^{+} \rightarrow \mathbb{R}^{+}$. The modified adaptive law is taken to be

$$
\begin{aligned}
\Xi_{D}\left(d_{\max }\right): u(t) & =-a x(t)-\hat{\theta}(t) \phi(x(t)) \\
\dot{\hat{\theta}} & =\alpha D_{\Omega_{0}\left(d_{\max }\right)}(x) x(t) \phi(x(t)) \quad \hat{\theta}(0)=0, \quad \eta_{0}=\frac{d_{\max }}{a}
\end{aligned}
$$

where $D_{\Phi}(x):=0$ if $x \in \Phi$ and $D_{\Phi}(x):=1$, elsewhere. We denote the respective closed loop system by $\left(\Sigma\left(x_{0}, \theta, d(\cdot)\right), \Xi_{D}\left(d_{\max }\right)\right)$. The following theorem establishes the properties of such controllers:

\section{Theorem 2.1}

Consider the closed loop system $\left(\Sigma\left(x_{0}, \theta, d(\cdot)\right), \Xi_{D}\left(d_{\max }\right)\right)$ defined by (1), (4), where $d(\cdot)$ is bounded. Assume that $d_{\max }$ is such that $\|d(\cdot)\|_{\mathscr{L}^{\infty}} \leqslant d_{\max }$. Then for any $x_{0} \in \mathbb{R}$, the following properties hold:

D1. There exist a unique solution $(x(\cdot), \hat{\theta}(\cdot)): \mathbb{R}^{+} \rightarrow \mathbb{R}^{2}$.

D2. $x(\cdot), u(\cdot), \hat{\theta}(\cdot)$ are uniformly bounded as a continuous function of $x_{0},|\theta|, d_{\max }$.

D3. $x(t) \rightarrow \Omega_{0}$ as $t \rightarrow \infty$. 
Proof

Due to the discontinuity in the right-hand side of the differential equations defining the deadzone controller (4), the solution of the closed loop is considered in Filippov's sense [10].

The proof of D1, D3 is standard and can be found e.g. in Reference [11], see also References $[3,5,6]$. An outline proof of $\mathrm{D} 2$ (which is directly required later in this paper) is as follows: Let $\tilde{\theta}(t):=\theta-\hat{\theta}(t)$, and define the Lyapunov function

$$
V(x(t), \tilde{\theta}(t))=\frac{1}{2} x(t)^{2}+\frac{1}{2 \alpha} \tilde{\theta}(t)^{2}
$$

A routine calculation shows $\dot{V}(x(t), \tilde{\theta}(t))=-a x(t)^{2}+x(t) d(t)$ for all $x \notin \Omega_{0}\left(d_{\max }\right)$. Considering the different situations of $x_{0}$ inside, outside, or on the boundary of the dead-zone $\Omega_{0}\left(d_{\max }\right)$, eventually yield $V(x(t), \tilde{\theta}(t)) \leqslant V_{0}\left(x_{0},|\theta|, d_{\max }\right)$ for all $t \geqslant 0$, where

$$
V_{0}\left(x_{0},|\theta|, d_{\max }\right):=\frac{1}{2} \max \left(x_{0}^{2}, \eta_{0}^{2}\right)+\frac{1}{2 \alpha} \theta^{2}
$$

From this and (4), (5) one can easily bound $x(\cdot), \hat{\theta}(\cdot)$ uniformly as continuous functions of $V_{0}:=V_{0}\left(x_{0},|\theta|, d_{\max }\right)$ :

$$
x(t) \leqslant \sqrt{2 V_{0}}, \quad|\hat{\theta}(t)| \leqslant \sqrt{2 \alpha V_{0}}
$$

Finally, the uniform boundedness of $u(\cdot)$ in terms of a continuous function of $V_{0}\left(x_{0},|\theta|, d_{\max }\right)$ follows from (3) and the continuity of $\phi(\cdot)$.

2.2.2. Projection modification. The projection modification [4] is an alternative method to eliminate parameter drift by keeping the parameter estimates within some a priori defined bounds $\Pi\left(\theta_{\max }\right)$ where $\theta_{\max }$ is the a priori knowledge of the parametric uncertainty level, and is defined as the strict upper bound of $|\theta|$. Consider the unmodified adaptive law $\hat{\theta}(t)=g(x(t), \hat{\theta}(t))$, $\hat{\theta}(0)=0$. By this method we project $g:=g(x(t), \hat{\theta}(t))$ on the hyperplane tangent to boundary $\partial \Pi\left(\theta_{\max }\right)$ at $\hat{\theta}(t)$ when $\hat{\theta}(t)$ is on the boundary $\partial \Pi\left(\theta_{\max }\right)$ and $g$ pointing outward. The general definition of the projection can be found in Reference [12]. For scalar systems where $\theta \in \mathbb{R}$, a simplified version of parameter projection can be obtained by defining $\Pi\left(\theta_{\max }\right):=\left[-\theta_{\max }, \theta_{\max }\right]$, and

$$
\operatorname{Proj}_{\Pi\left(\theta_{\max }\right)}(g, \hat{\theta})= \begin{cases}g, & |\hat{\theta}|<\theta_{\max } \text { or } \hat{\theta} g \leqslant 0 \\ 0, & |\hat{\theta}|=\theta_{\max } \text { and } \hat{\theta} g>0\end{cases}
$$

The modified adaptive law is taken to be

$$
\dot{\hat{\theta}}(t)=\operatorname{Proj}_{\Pi\left(\theta_{\max }\right)}(g, \hat{\theta}), \quad \hat{\theta}(0)=0
$$

Consequently, the projection controller $\Xi_{P}\left(\theta_{\max }\right)$ for systems (1) is defined as follows:

$$
\begin{aligned}
\Xi_{P}\left(\theta_{\max }\right): u(t) & =-a x(t)-\hat{\theta}(t) \phi(x(t)) \\
\dot{\hat{\theta}}(t) & =\operatorname{Proj}_{\Pi\left(\theta_{\max }\right)}(\alpha x(t) \phi(x(t))), \quad \hat{\theta}(0)=0
\end{aligned}
$$

\footnotetext{
Such a dead-zone has been chosen for simplicity, however, in practical situations, principled approaches to avoiding chattering are available, see for example the hysteresis dead-zone approach of Reference [8]-where analogous results to those presented in this paper for the standard dead-zone can be found.
} 
The robustness of the respective closed loop system $\left(\Sigma\left(x_{0}, \theta, d(\cdot)\right), \Xi_{P}\left(\theta_{\max }\right)\right)$ is given by the following theorem:

\section{Theorem 2.2}

Consider the closed loop $\left(\Sigma\left(x_{0}, \theta, d(\cdot)\right), \Xi_{P}\left(\theta_{\max }\right)\right)$ defined by (1), (10). Assume $\theta_{\max }$ is such that $|\theta| \leqslant \theta_{\max }$. Then, for any $x_{0} \in \mathbb{R}$ :

P1. The solution $(x(\cdot), \hat{\theta}(\cdot)): \mathbb{R}^{+} \rightarrow \mathbb{R}^{2}$ exists.

P2. $x(\cdot), u(\cdot), \hat{\theta}(\cdot)$ are uniformly bounded as a continuous function of $x_{0},\|d\|, \theta_{\max }$.

\section{Proof}

Since the right-hand side of the closed loop $\left(\Sigma\left(x_{0}, \theta, d(\cdot)\right), \Xi_{P}\left(\theta_{\max }\right)\right)$ is locally Lipschitz, an absolutely continuous global solution $(x(\cdot), \hat{\theta}(\cdot))$ exists once the boundedness of solution has been shown. Let $\tilde{\theta}(t)=\theta-\hat{\theta}(t)$, and define the same Lyapunov function as (5). By a well-known property of the projection operator $-\tilde{\theta}(t) \operatorname{Proj}(x(t) \phi(x)) \leqslant-\tilde{\theta}(t) x(t) \phi(x)$ (see e.g. Reference [12]), a routine calculation shows

$$
\dot{V}(x(t), \tilde{\theta}(t)) \leqslant-k\left(V(x(t), \tilde{\theta}(t))-V^{*}\left(\theta_{\max },\|d\|\right)\right)
$$

where

$$
V^{*}\left(\theta_{\max },\|d\|\right):=\frac{1}{2} \theta_{\max }^{2}+\frac{\|d\|^{2}}{2 k a}
$$

and $0<k<a / 2$. It follows that $\dot{V}(x(t), \tilde{\theta}(t)) \leqslant 0$ for all $V \geqslant V^{*}$. Therefore,

$$
V(x(t), \tilde{\theta}(t)) \leqslant V_{0}^{\prime}\left(x_{0},\|d\|, \theta_{\max }\right):=\max \left\{V\left(x_{0}, 0\right), V^{*}\left(\theta_{\max },\|d\|\right)\right\} \quad \forall t \geqslant 0
$$

The uniform boundedness of $x(\cdot), u(\cdot)$ as a continuous function of $V_{0}^{\prime}\left(x_{0},\|d\|, \theta_{\max }\right)$ follows from (10), (13) and continuity of $\phi(\cdot)$.

\subsection{Statement of the main results}

The ultimate goal in control theory is to design control laws which achieve good performance for any member of a specified class of systems. Consider a system $\Sigma$ which belongs to the set of all admissible systems $\mathscr{S}^{*}$. The performance of a controller $\Xi$ is given by a cost functional $J$ of some measurable signals (state/output/input). The goal of this paper is to establish a comparison between dead-zone and projection methods. We are interested in a worst case scenario, i.e. a performance $\mathscr{P}$ which is defined over the power set of $\mathscr{S}^{*}$ and is formulated as a supremum of all cost functionals. Furthermore, the performance measure will be non-singular, i.e. penalize both the state $(x)$ and the input $(u)$ of the plant; specifically, we will consider cost functionals of the form

$$
\mathscr{P}\left(\Sigma\left(\mathscr{X}_{0}(\gamma), \Delta(\delta), \mathscr{D}(\varepsilon)\right), \Xi\right)=\sup _{x_{0} \in \mathscr{X}_{0}(\gamma)} \sup _{\theta \in \Delta(\delta)} \sup _{d \in \mathscr{D}(\varepsilon)}\left(\|x(\cdot)\|_{\mathscr{L}^{\infty}}+\|u(\cdot)\|_{\mathscr{L}^{\infty}}+\|\dot{u}(\cdot)\|_{\mathscr{L}^{\infty}}\right)
$$


where

$$
\begin{aligned}
\mathscr{D}(\varepsilon) & :=\left\{d(\cdot) \in \mathscr{L}^{\infty} \mid\|d(\cdot)\|_{\mathscr{L}^{\infty}} \leqslant \varepsilon\right\} \\
\Delta(\delta) & :=\{\theta \in \mathbb{R}|| \theta \mid \leqslant \delta\} \\
\mathscr{X}_{0}(\gamma) & :=\left\{x_{0} \in \mathbb{R} \mid\left\|x_{0}\right\| \leqslant \gamma\right\}
\end{aligned}
$$

for some $\varepsilon, \delta \geqslant 0$ and $\gamma>0$.

The following theorems are the main results of the paper:

\section{Theorem I}

Suppose $\phi(\cdot)$ satisfies conditions 2-a-2-c. Consider the system $\Sigma\left(x_{0}, \theta, d(\cdot)\right)$ and the controllers $\Xi_{D}\left(d_{\max }\right)$ and $\Xi_{P}\left(\theta_{\max }\right)$ defined by (1), (4) and (10), respectively. Consider the transient performance cost functional (14). Then for all $d_{\max } \geqslant \varepsilon$, there exists $\theta_{\max }^{*} \geqslant \delta$ such that for all $\theta_{\max } \geqslant \theta_{\max }^{*}$,

$$
\mathscr{P}\left(\Sigma\left(\mathscr{X}_{0}(\gamma), \Delta(\delta), \mathscr{D}(\varepsilon)\right), \Xi_{P}\left(\theta_{\max }\right)\right)>\mathscr{P}\left(\Sigma\left(\mathscr{X}_{0}(\gamma), \Delta(\delta), \mathscr{D}(\varepsilon)\right), \Xi_{D}\left(d_{\max }\right)\right)
$$

This theorem can be interpreted as stating that if the a priori knowledge of the parametric uncertainty level $\theta_{\max }$ is sufficiently conservative $\left(\theta_{\max } \geqslant \theta_{\max }^{*}\right)$, then the dead-zone based design will outperform the projection based design.
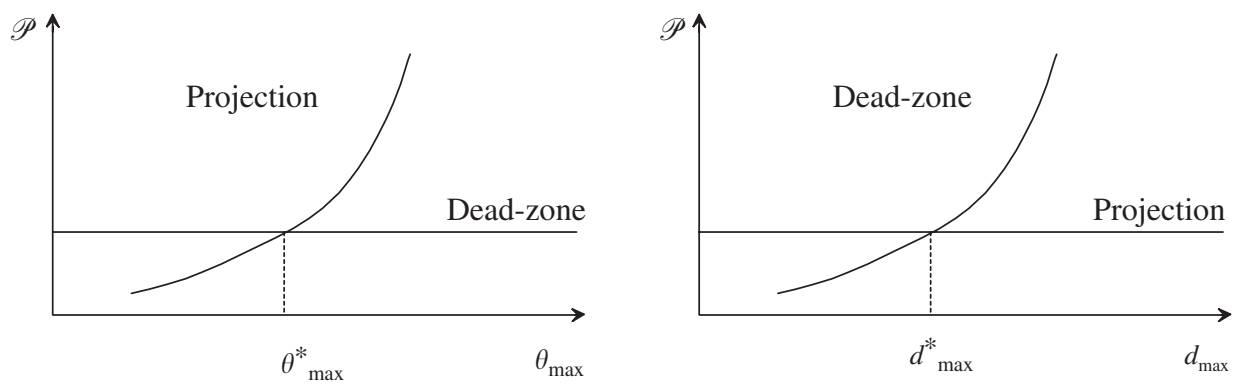

Figure 1. Statement of the main results: Theorem I (left), Theorem II (right).

\section{Theorem II}

Suppose $\phi(\cdot)$ satisfies conditions 2-c. Consider the system $\Sigma\left(x_{0}, \theta, d(\cdot)\right)$ and the controllers $\Xi_{D}\left(d_{\max }\right)$ and $\Xi_{P}\left(\theta_{\max }\right)$ defined by (1), (4) and (10), respectively. Consider the transient performance cost functional (14). Then there exists $\delta>0$ such that for all $\theta_{\max } \geqslant \delta$, there exists $d_{\max }^{*} \geqslant \varepsilon$ so that for all $d_{\max } \geqslant d_{\max }^{*}$,

$$
\mathscr{P}\left(\Sigma\left(\mathscr{X}_{0}(\gamma), \Delta(\delta), \mathscr{D}(\varepsilon)\right), \Xi_{D}\left(d_{\max }\right)\right)>\mathscr{P}\left(\Sigma\left(\mathscr{X}_{0}(\gamma), \Delta(\delta), \mathscr{D}(\varepsilon)\right), \Xi_{P}\left(\theta_{\max }\right)\right)
$$

This theorem can be interpreted as stating that above a certain uncertainty level $\delta$, if the a priori knowledge $d_{\max }$ of the disturbance level is sufficiently conservative ( $d_{\max } \geqslant d_{\max }^{*}$ ), then the projection design will outperform the dead-zone design.

In fact, as it has been shown in Figure 1, we will prove the stronger results that the ratio between the two costs can be made arbitrarily large (Figure 1). That is, for Theorem I:

$$
\frac{\mathscr{P}\left(\Sigma\left(\mathscr{X}_{0}(\gamma), \Delta(\delta), \mathscr{D}(\varepsilon)\right), \Xi_{P}\left(\theta_{\max }\right)\right)}{\mathscr{P}\left(\Sigma\left(\mathscr{X}_{0}(\gamma), \Delta(\delta), \mathscr{D}(\varepsilon)\right), \Xi_{D}\left(d_{\max }\right)\right)} \rightarrow \infty \text { as } \theta_{\max } \rightarrow \infty \quad \forall d_{\max } \geqslant \varepsilon
$$


Alternatively, for Theorem II, we have

$$
\frac{\mathscr{P}\left(\Sigma\left(\mathscr{X}_{0}(\gamma), \Delta(\delta), \mathscr{D}(\varepsilon)\right), \Xi_{D}\left(d_{\max }\right)\right)}{\mathscr{P}\left(\Sigma\left(\mathscr{X}_{0}(\gamma), \Delta(\delta), \mathscr{D}(\varepsilon)\right), \Xi_{P}\left(\theta_{\max }\right)\right)} \rightarrow \infty \text { as } d_{\max } \rightarrow \infty \quad \forall \theta_{\max } \geqslant \delta
$$

Note that if appropriate sign changes are made to the controller, equivalent results also hold if the sign is changed in condition 2-b, i.e. if $\partial \phi(x) /\left.\partial x\right|_{x=0}<0$.

\section{PROOF OF THEOREM I}

Firstly, we show that $\mathscr{P}=\infty$ for the unmodified design (3) (Proposition 3.3). From this we can show that the projection modification design, $\Xi_{P}\left(\theta_{\max }\right)(10)$ has the property that $\mathscr{P} \rightarrow \infty$ as $\theta_{\max } \rightarrow \infty$ (Proposition 3.4). Finally, we show that $\mathscr{P}<\infty$ for the dead-zone design, $\Xi_{D}\left(d_{\max }\right)$ (4) and that $\mathscr{P}$ is independent of $\theta_{\max }$ (Propositions 3.5). This suffices to establish Theorem I.

\section{Proposition 3.1}

Suppose $\phi(\cdot)$ satisfies conditions 2-a-2-c. Consider the closed loop system $\left(\Sigma\left(x_{0}, \theta, d(\cdot)\right), \Xi\right)$ defined by (1), (3), where $d(t)=\varepsilon$, for some $\varepsilon \neq 0$. Then

$$
x(t) \rightarrow 0 \text { as } t \rightarrow \infty \Leftrightarrow \hat{\theta}(t) \rightarrow \infty \text { as } t \rightarrow \infty
$$

Proof

$\rightarrow)$ Suppose for contradiction $\hat{\theta}(t) \nrightarrow \infty$ as $t \rightarrow \infty$. Then $\hat{\theta}(t) \rightarrow \hat{\theta}^{*}<\infty$, since $\hat{\theta}(t)$ is monotonically increasing by (2). Therefore by continuity of $(1),(x(t), \hat{\theta}(t))=\left(0, \hat{\theta}^{*}\right)$ is an equilibrium point of the closed loop $\left(\Sigma\left(x_{0}, \theta, d(\cdot)\right)\right.$, $\left.\Xi\right)$. Hence $\left(0, \hat{\theta}^{*}\right)$ must be a solution of the following equations:

$$
\begin{array}{r}
-a x(t)+(\theta-\hat{\theta}(t)) \phi(x(t))+\varepsilon=0 \\
\alpha x(t) \phi(x(t))=0
\end{array}
$$

Clearly, given $\varepsilon \neq 0$ and assumption 2-a, (21) has no solution, hence contradiction. Therefore, $\hat{\theta}(t) \rightarrow \infty$ as $t \rightarrow \infty$.

$\leftarrow)$ Defining the Lyapunov function $V(x(t))=x(t)^{2} / 2$, we have that

$$
\dot{V}(x(t))=-a x(t)^{2}+\varepsilon x(t)+\varphi(t)
$$

where in the interest of brevity we have denoted

$$
\varphi(t):=\varphi(x, \phi, \hat{\theta})=(\theta-\hat{\theta}(t)) x(t) \phi(x(t))
$$

It follows that $V(x(t))$ is decreasing if

$$
a x(t)^{2} / 2-\varphi(t) \geqslant \varepsilon^{2} / 2 a
$$


Now, we claim the convergence of $x(\cdot)$ : if $x(t) \nrightarrow 0$ as $t \rightarrow \infty$ then either $1 . \liminf _{t \rightarrow \infty}$ $|x(t)|>0$ or $2 . \liminf \inf _{t \rightarrow \infty}|x(t)|=0$ :

1. Suppose $\liminf \operatorname{in}_{t \rightarrow \infty}|x(t)|>0$. Then there exists $\varepsilon^{\prime}>0 \quad$ s.t. $|x(t)|>\varepsilon^{\prime}$ for all $t$. Observing that conditions 2-a and 2-b imply that $x \phi(x)>0$ for all $x \neq 0$, it follows by (23) and 2-c that $\varphi(t) \rightarrow-\infty$ as $\hat{\theta}(t) \rightarrow \infty$. Hence by (22), $\dot{V}(x(t)) \rightarrow-\infty$ as $t \rightarrow \infty$, i.e. $V(x(t)) \rightarrow-\infty$. This contradicts the positive definiteness of $V(\cdot)$.

2. If $\lim _{\inf _{t \rightarrow \infty}}|x(t)|=0$, then there exists $\varepsilon^{\prime}>0$ and a positive divergent sequence $\left\{t_{k}\right\}_{k \geqslant 1}$ such that $\dot{V}\left(x\left(t_{k}\right)\right)>0$ and $\left|x\left(t_{k}\right)\right|>\varepsilon^{\prime}$. Since by (23), $\varphi\left(t_{k}\right) \rightarrow-\infty$ as $k \rightarrow \infty$, it follows that (24) holds at time $t_{k}$, hence contradiction.

Therefore, $x(t) \rightarrow 0$ as $t \rightarrow \infty$.

Proposition 3.2

Suppose $\phi(\cdot)$ satisfies conditions 2-a-2-c. Consider the closed loop system $\left(\Sigma\left(x_{0}, \theta, d(\cdot)\right), \Xi\right)$ defined by (1), (3), where $d(t)=\varepsilon$, for some $\varepsilon \neq 0$. If $x(t)$ is bounded and uniformly continuous, then

$$
x(t) \rightarrow 0, \quad \hat{\theta}(t) \rightarrow \infty \text { as } t \rightarrow \infty
$$

Proof

Suppose for contradiction $x(t) \nrightarrow 0$ as $t \rightarrow \infty$. Then there exists a $M>0$ and a positive divergent sequence $\left\{t_{k}\right\}_{k \geqslant 1}$ for which $x\left(t_{k}\right) \geqslant M$. Since, by assumption, $x(t)$ is uniformly continuous, then, for $\varepsilon=M / 2$ we have

$$
\exists \omega>0 \text { s.t. } \forall \tau \in[0, \omega], \quad \forall t>0 \quad|x(t)-x(t+\tau)|<\frac{M}{2}
$$

Therefore, $\left|x\left(t_{k}\right)-x\left(t_{k}+\tau\right)\right|<M / 2$ and since $x\left(t_{k}\right) \geqslant M$, we have that $x\left(t_{k}+\tau\right)>M / 2$, i.e. $x(t) \geqslant M / 2$ for all $t \in\left[t_{k}, t_{k}+\omega\right]$. Now by (2), the boundedness of $x(\cdot)$, and the continuity of $\phi(\cdot)$, we have that $\phi(x(t)) \geqslant \beta>0$ for some $\beta$, i.e.

$$
\exists N>0 \text { s.t. } \alpha x(t) \phi(x(t)) \geqslant N \quad \forall t \in\left[t_{k}, t_{k}+\delta\right]
$$

It follows that

$$
\int_{t_{k}}^{t_{k}+\delta} \alpha x(\tau) \phi(x(\tau)) \mathrm{d} \tau \geqslant N \delta
$$

With no loss of generality, we may assume $t_{k+1}-t_{k} \geqslant \omega$. It follows that

$$
\hat{\theta}\left(t_{k}+\omega\right)=\int_{0}^{t_{k}+\omega} \dot{\hat{\theta}}(\tau) \mathrm{d} \tau=\int_{0}^{t_{k}+\omega} \alpha x(\tau) \phi(x(\tau)) \mathrm{d} \tau \geqslant k N \delta
$$

so $\hat{\theta}\left(t_{k}+\omega\right) \rightarrow \infty$ as $k \rightarrow \infty$, hence $\hat{\theta}(t) \rightarrow \infty$ as $t \rightarrow \infty$. It follows by Proposition 3.1 that $x(t) \rightarrow 0$ as $t \rightarrow \infty$, hence contradiction. From this and Proposition 3.1, the claim of the proposition follows. 
Proposition 3.3

Suppose $\phi(\cdot)$ satisfies conditions 2-a-2-c. Consider the closed loop $\left(\Sigma\left(x_{0}, \theta, d(\cdot)\right), \Xi\right)$ defined by Equations (1) and (3) and the transient performance cost functional (14). Then

$$
\mathscr{P}\left(\Sigma\left(\mathscr{X}_{0}(\gamma), \Delta(\delta), \mathscr{D}(\varepsilon)\right), \Xi\right)=\infty
$$

\section{Proof}

Let $x_{0} \in \mathscr{X}_{0}(\gamma), \theta \in \Delta(\delta)$, and choose $d(t)=\varepsilon \neq 0$. Denote lim $\sup _{t \rightarrow \infty}$ by $\overline{\lim }$. Suppose for contradiction $\mathscr{P}\left(\Sigma\left(x_{0}, \theta, d(\cdot)\right), \Xi\right)<\infty$. Consider $\dot{x}(t)$. There are two cases: either

1. $\varlimsup \lim |\dot{x}(t)|=\infty$ or $2 . \overline{\lim }|\dot{x}(t)|<\infty$ :

(1) Suppose $\varlimsup|\dot{\lim }| \dot{x}(t) \mid=\infty$, i.e. $\overline{\lim }|-a x(t)+(\theta-\hat{\theta}(t)) \phi(x(t))+\varepsilon|=\infty$. Therefore, either

(a) $\varlimsup|x(t)|=\infty$, which implies that $\|x(\cdot)\|_{\mathscr{L}^{\infty}}=\infty$, hence contradiction, or

(b) $\varlimsup|x(t)|<\infty$, therefore $\varlimsup \lim |u(t)|=\infty$, i.e. $\|u(\cdot)\|_{\mathscr{L}^{\infty}}=\infty$, hence contradiction.

(2) Suppose $\overline{\lim }|\dot{x}(t)|<\infty$ i.e. $x(t)$ is uniformly continuous. Again there are two possibilities: either a) $\varlimsup|x(t)|=\infty$, or b) $\varlimsup|\lim | x(t) \mid<\infty$ :

(a) Suppose $\varlimsup|x(t)|=\infty$, which implies that $\|x(\cdot)\|_{\mathscr{L}^{\infty}}=\infty$, hence contradiction.

(b) Suppose $\varlimsup|x(t)|<\infty$, i.e. $x(t)$ is bounded. Therefore, by Proposition 3.2

$$
x(t) \rightarrow 0, \quad \hat{\theta}(t) \rightarrow \infty \text { as } t \rightarrow \infty
$$

Considering $\varlimsup \dot{\lim } \dot{u}(t)$, by applying (31), we observe that

$$
\begin{aligned}
& \varlimsup \dot{\lim }(t)=\varlimsup\left[(-a x(t)+(\theta-\hat{\theta}(t)) \phi(x(t))+\varepsilon)\left(-a+\hat{\theta}(t) \frac{\partial \phi(x)}{\partial x}\right)\right. \\
& \left.+x(t) \phi(x(t))^{2}\right] \\
& =\varlimsup\left[-(\hat{\theta}(t) \phi(x(t))-\varepsilon)\left(a+\hat{\theta}(t) \frac{\partial \phi(x)}{\partial x}\right)\right]
\end{aligned}
$$

Now there are two possibilities: either (i) $\hat{\theta}(t) \phi(x(t)) \nrightarrow \varepsilon$ (including the possibility that $\lim _{t \rightarrow \infty} \hat{\theta}(t) \phi(x(t))$ does not exist), or (ii) $\lim _{t \rightarrow \infty} \hat{\theta}(t) \phi(x(t))=\varepsilon$ :

(i) Suppose $\lim _{t \rightarrow \infty} \hat{\theta}(t) \phi(x(t))$ does not exist or $\hat{\theta}(t) \phi(x(t)) \nrightarrow \varepsilon$ as $t \rightarrow \infty$. Since

$$
a+\hat{\theta}(t) \frac{\partial \phi(x)}{\partial x} \rightarrow \infty \text { as } x(t) \rightarrow 0, \quad \hat{\theta}(t) \rightarrow \infty
$$

it follows by (2-b) that $\|\dot{u}(\cdot)\|_{\mathscr{L}^{\infty}}=\infty$; hence contradiction.

(ii) Suppose $\lim _{t \rightarrow \infty} \hat{\theta}(t) \phi(x(t))=\varepsilon$. By (31) we have that

$$
\forall \hat{\theta}^{*}>0 \quad \exists T>0 \text { s.t. } \hat{\theta}(t)>\hat{\theta}^{*} \quad \forall t>T .
$$

Now we choose $d_{2}(\cdot)$ as follows

$$
d_{2}(t)= \begin{cases}\varepsilon, & t \leqslant T, \\ -\varepsilon, & t>T\end{cases}
$$

Note that $d_{2}(t)=d(t)$ for all $t \leqslant T$. With this choice, by smoothness of $\phi(\cdot)$, continuity of $x(\cdot)$ and causality, we have that

$$
\lim _{t \rightarrow T^{+}} x(t)=x(T), \quad \lim _{t \rightarrow T^{+}} \hat{\theta}(t)=\hat{\theta}(T)
$$


where $\lim _{t \rightarrow T^{+}}$denote $\lim _{t \rightarrow T, t>T}$. It follows that

$$
\left(\lim _{t \rightarrow T^{+}} \dot{u}(t)\right)-\dot{u}(T)=2 \varepsilon\left(a+\hat{\theta}(t) \frac{\partial \phi(x)}{\partial x}\right) \geqslant 2 \hat{\theta}^{*} \frac{\partial \phi(x)}{\partial x} \varepsilon
$$

By assumption 2-b, difference (37) can be made arbitrarily large by choosing a suitable $\hat{\theta}^{*}$. Then either $\dot{u}(T)$ is large or $\lim _{t \rightarrow T^{+}} \dot{u}(t)$ is large. Therefore, $\|\dot{u}(\cdot)\|_{\mathscr{L}^{\infty}}$ can be made arbitrarily large; hence contradiction.

The proof is completed since $\mathscr{P}\left(\Sigma\left(\mathscr{X}_{0}(\gamma), \Delta(\delta), \mathscr{D}(\varepsilon)\right), \Xi\right) \geqslant \mathscr{P}\left(\Sigma\left(x_{0}, \theta, d(\cdot)\right), \Xi\right)=\infty$.

\section{Proposition 3.4}

Suppose $\phi(\cdot)$ satisfies conditions 2-a-2-c. Consider the closed loop system $\left(\Sigma\left(x_{0}, \theta, d(\cdot)\right)\right.$, $\left.\Xi_{P}\left(\theta_{\max }\right)\right)$ defined by Equations (1) and (10). Then

$$
\mathscr{P}\left(\Sigma\left(\mathscr{X}_{0}(\gamma), \Delta(\delta), \mathscr{D}(\varepsilon)\right), \Xi_{P}\left(\theta_{\max }\right)\right) \rightarrow \infty \quad \text { as } \theta_{\max } \rightarrow \infty
$$

\section{Proof}

It is convenient to define

$$
\mathscr{P}_{[0, T]}(\Sigma, \Xi)=\left(\|x(\cdot)\|_{\mathscr{L}^{\infty}[0, T]}+\|u(\cdot)\|_{\mathscr{L}^{\infty}[0, T]}+\|\dot{u}(\cdot)\|_{\mathscr{L}^{\infty}[0, T]}\right)
$$

Now let $M>0$. By Proposition 3.3, there exists $x_{0} \in \mathscr{X}_{0}, d(\cdot) \in \mathscr{D}(\varepsilon), \theta \in \Delta(\delta)$ so that

$$
\mathscr{P}_{[0, \infty)}\left(\Sigma\left(x_{0}, \theta, d(\cdot)\right), \Xi\right) \geqslant 2 M
$$

It follows that there exists $T>0$ s.t. $\mathscr{P}_{[0, T]}\left(\Sigma\left(x_{0}, \theta, d(\cdot)\right), \Xi\right) \geqslant M$. Since $\theta_{\max }$ diverges, by choosing $\theta_{\max }=2 \hat{\theta}(T)$, we have that $\theta_{\max }>\hat{\theta}(T)$, i.e. the unmodified and the projection designs are identical on $[0, T]$. Therefore,

$$
\mathscr{P}\left(\Sigma\left(\mathscr{X}_{0}(\gamma), \Delta(\delta), \mathscr{D}(\varepsilon)\right), \Xi_{P}\left(\theta_{\max }\right)\right) \geqslant \mathscr{P}_{[0, T]}\left(\Sigma\left(x_{0}, \theta, d(\cdot)\right), \Xi_{P}\left(\theta_{\max }\right)\right) \geqslant M
$$

Since this holds for all $M>0$, this completes the proof.

\section{Proposition 3.5}

The closed loop $\left(\Sigma\left(x_{0}, \theta, d(\cdot)\right), \Xi_{D}\left(d_{\max }\right)\right)$ defined by (1) and (4) has the property

$$
\mathscr{P}\left(\Sigma\left(\mathscr{X}_{0}(\gamma), \Delta(\delta), \mathscr{D}(\varepsilon)\right), \Xi_{D}\left(d_{\max }\right)\right)<\infty \quad \forall d_{\max }>\varepsilon
$$

\section{Proof}

Let $x_{0} \in \mathscr{X}_{0}(\gamma), \theta \in \Delta(\delta)$ and $d \in \mathscr{D}(\varepsilon)$. The uniform boundedness of signals $x(\cdot), \hat{\theta}(\cdot), u(\cdot)$ as a continuous function of $V_{0}\left(x_{0},|\theta|, d_{\mathrm{max}}\right)$ follow from Theorem 2.1. Therefore by $(1), \dot{x}(\cdot)$ is uniformly bounded in terms of a continuous function of $V_{0}\left(x_{0},|\theta|, d_{\max }\right)$. So

$$
\dot{u}(t)=-a \dot{x}(t)-\hat{\theta}(t) \frac{\partial \phi(x)}{\partial x} \dot{x}(t)-\alpha D_{\Omega_{0}} x(t) \phi(x(t))^{2}
$$

is uniformly bounded as a continuous function of $V_{0}\left(x_{0},|\theta|, d_{\max }\right)$. That is

$$
\mathscr{P}\left(\Sigma\left(x_{0}, \theta, d(\cdot)\right), \Xi_{D}\left(d_{\max }\right)\right) \leqslant M\left(V_{0}\left(x_{0},|\theta|, d_{\max }\right)\right)
$$


where $M(\cdot)$ is continuous. The proof is completed by taking the supremum over system arguments $x_{0}, \theta, d$, hence

$$
\mathscr{P}\left(\Sigma\left(\mathscr{X}_{0}(\gamma), \Delta(\delta), \mathscr{D}(\varepsilon)\right), \Xi_{D}\left(d_{\max }\right)\right)<\infty \quad \forall d_{\max }>\varepsilon
$$

\section{Proof of Theorem I}

This is a simple consequence of Propositions 3.4 and 3.5.

\section{PROOF OF THEOREM II}

In order to prove Theorem II, first we give the following propositions:

\section{Proposition 4.1}

Suppose $\phi(\cdot)$ satisfies condition 2-c. Consider the closed loop system $\left(\Sigma\left(x_{0}, \theta, d(\cdot)\right), \Xi_{D}\left(d_{\max }\right)\right)$ defined by (1), (4). Then $\exists \delta>0$ such that

$$
\mathscr{P}\left(\Sigma\left(\mathscr{X}_{0}(\gamma), \Delta(\delta), \mathscr{D}(\varepsilon)\right), \Xi_{D}\left(d_{\max }\right)\right) \rightarrow \infty \quad \text { as } d_{\max } \rightarrow \infty
$$

\section{Proof}

Note that by (4), $\left\|\Omega_{0}\right\| \rightarrow \infty$ as $d_{\max } \rightarrow \infty$ (c.f. to the discussion in Section 4.1). Let $x_{0} \in \mathscr{X}_{0}(\gamma)$, $\theta \in \Delta(\delta)$ and $d \in \mathscr{D}(\varepsilon)$. Suppose $x_{0} \in \Omega_{0}$ (i.e. $\gamma<\eta_{0}$ ). We define $\tau$ as follows:

$$
\tau= \begin{cases}\infty & \text { if } x(t) \in \Omega_{0} \forall t \geqslant 0 \\ \inf \left\{t \geqslant 0|| x(t) \mid=\eta_{0}\right\} & \text { otherwise }\end{cases}
$$

Note that by dead-zone definition $(4), \dot{\hat{\theta}}(t)=0$ for all $t \in[0, \tau)$, hence $\hat{\theta}(t)=0$ for all $t \in[0, \tau)$ since $\hat{\theta}(0)=0$. Therefore,

$$
\dot{x}(t)=-a x(t)+\theta \phi(x(t))+d(t) \quad \forall t \in[0, \tau)
$$

Condition 2-c can be rewritten in the following form:

$$
\sup _{x \in \mathbb{R}}\left|\frac{x}{\phi(x)}\right| \leqslant M<\infty
$$

It follows that there exists $\delta=2 a M$ such that if $\theta=\delta$, then $\forall x(t)>0$,

$$
-a x+\theta \phi(x)=\left(-a \frac{x}{\phi(x)}+\theta\right) \phi(x)=\left(-a \frac{|x|}{|\phi(x)|}+\theta\right)|\phi(x)| \geqslant a M|\phi(x)|>0
$$

It follows that if $d(t)=\varepsilon$, then $|\dot{x}(t)|>\varepsilon$ for all $t \in[0, \tau)$ i.e. the trajectory $x(t)$ hits the boundary $\partial \Omega_{0}$ in finite time, hence $\tau<\infty$. It follows that

$$
\|x(\cdot)\|_{\mathscr{L}^{\infty}} \geqslant|x(\tau)|=\left|\partial \Omega_{0}\right|
$$

If $x_{0} \notin \Omega_{0}$ then we are outside the dead-zone, i.e. ||$x(\cdot)\left|\mathscr{L}_{\infty} \geqslant\right| x_{0}|\geqslant| \partial \Omega_{0} \mid$. The proof is completed by taking $d_{\max } \rightarrow \infty$, i.e. $\left|\Omega_{0}\right| \rightarrow \infty$. Hence,

$$
\mathscr{P}\left(\Sigma\left(\mathscr{X}_{0}(\gamma), \Delta(\delta), \mathscr{D}(\varepsilon)\right), \Xi_{D}\left(d_{\max }\right)\right) \geqslant \mathscr{P}\left(\Sigma\left(x_{0}, \theta, d(\cdot)\right), \Xi_{D}\left(d_{\max }\right)\right)=\infty
$$




\section{Proposition 4.2}

Consider the closed loop system $\left(\Sigma\left(x_{0}, \theta, d(\cdot)\right), \Xi_{P}\left(\theta_{\max }\right)\right)$ defined by (1), (10). Consider the transient performance cost functional defined in (14). Then

$$
\mathscr{P}\left(\Sigma\left(\mathscr{X}_{0}(\gamma), \Delta(\delta), \mathscr{D}(\varepsilon)\right), \Xi_{P}\left(\theta_{\max }\right)\right)<\infty \quad \forall \theta_{\max } \geqslant \delta
$$

\section{Proof}

Let $x_{0} \in \mathscr{X}_{0}(\gamma), \theta \in \Delta(\delta)$ and $d \in \mathscr{D}(\varepsilon)$. A direct application of property P2 of Theorem 2.2 guarantees the uniform boundedness of signals $x(\cdot), \hat{\theta}(\cdot), u(\cdot)$ of the closed-loop system $\left(\Sigma\left(x_{0}, \theta, d(\cdot)\right), \Xi_{P}\left(\theta_{\max }\right)\right)$ as a continuous function of $x_{0},\|d\|, \theta_{\max }$. Therefore by $(1), \dot{x}(\cdot)$ is uniformly bounded in terms of a continuous function of $x_{0},\|d\|, \theta_{\max }$. Hence

$$
\dot{u}(t)=-\left(a+\hat{\theta} \frac{\partial \phi(x)}{\partial x}\right) \dot{x}(t)-\alpha \phi(x(t)) \operatorname{Proj}(x(t) \phi(x(t)))
$$

is uniformly bounded in terms of a continuous function of $x_{0},\|d\|, \theta_{\max }$. It follows that there exists a continuous function $M\left(x_{0},\|d\|, \theta_{\max }\right)$, such that

$$
\mathscr{P}\left(\Sigma\left(x_{0}, \theta, d(\cdot)\right), \Xi_{P}\left(\theta_{\max }\right)\right) \leqslant M\left(x_{0},\|d\|, \theta_{\max }\right)<\infty
$$

The claim of proposition follows by taking the supremum over system parameters $x_{0}, \theta, d$ as in (15) on both sides of (55) and observing that, by uniform boundedness, the right-hand side remains bounded for all $\theta_{\max } \geqslant \delta$.

\section{Proof of Theorem II}

This is a simple consequence of Propositions 4.1 and 4.2.

The proof of above theorem is heavily based on the very natural assumption that the size of the dead-zone is a divergent function of a priori information on the disturbance level. In particular, $\eta_{0}:=\varrho\left(d_{\max }\right)=d_{\max } / a$ implies that $\mathscr{P}\left(\Sigma\left(\mathscr{X}_{0}(\gamma), \Delta(\delta), \mathscr{D}(\varepsilon)\right), \Xi_{D}\left(d_{\max }\right)\right) \rightarrow \infty$ as $d_{\max } \rightarrow$ $\infty$. In the following section we show that the other choices of $\eta_{0}$ also yield the similar results.

\subsection{Choices of dead-zone}

In this section, we consider alternative choices for the dead-zone, and variations on the definition of the controller. For brevity, only an outline of the relevant proofs are discussed, see Reference [8] for a fuller discussion. Let $\Omega_{0}:=\left[-\eta_{0}, \eta_{0}\right]$, where $\eta_{0}:=\varrho\left(d_{\max }\right)$, and define $\mathscr{P}:=$ $\mathscr{P}\left(\Sigma\left(\mathscr{X}_{0}(\gamma), \Delta(\delta), \mathscr{D}(\varepsilon)\right), \Xi\right)$ as in $(14)$. There are three distinct possibilities for $\eta_{0}:=\varrho\left(d_{\max }\right)^{\S}$

(i) $\eta_{0}:=\varrho\left(d_{\max }\right) \rightarrow \infty$ as $d_{\max } \rightarrow \infty$. It is straightforward to observe that $\mathscr{P}=\infty$ by Proposition 4.1.

(ii) $\eta_{0}:=\varrho\left(d_{\max }\right) \rightarrow 0$ as $d_{\max } \rightarrow \infty$. By shrinking the dead-zone, we have a sequence of modified controllers $\Xi_{D}\left(d_{\max }\right)$ tending to unmodified controller $\Xi$. It follows that as $d_{\max } \rightarrow \infty$, the performance of the sequence of modified closed loops $\mathscr{P}\left(\Sigma\left(\mathscr{X}_{0}(\gamma), \Delta(\delta)\right.\right.$, $\left.\mathscr{D}(\varepsilon)), \Xi_{D}\left(d_{\max }\right)\right)$ tends to the performance of that of unmodified closed loop

\footnotetext{
$\overline{{ }_{\S} \text { Other cases such }}$ as oscillatory but bounded $\varrho(\cdot)$ can be handled suitably by considering monotonic subsequences.
} 
$\mathscr{P}\left(\Sigma\left(\mathscr{X}_{0}(\gamma), \Delta(\delta), \mathscr{D}(\varepsilon)\right), \Xi\right)$ for which by Proposition $3.3, \mathscr{P}=\infty$, therefore,

$$
\mathscr{P}\left(\Sigma\left(\mathscr{X}_{0}(\gamma), \Delta(\delta), \mathscr{D}(\varepsilon)\right), \Xi_{D}\left(d_{\max }\right)\right) \rightarrow \infty \quad \text { as } d_{\max } \rightarrow \infty
$$

(iii) $\eta_{0}:=\varrho\left(d_{\max }\right) \leqslant c$ as $d_{\max } \rightarrow \infty$. Recall the closed loop $\left(\Sigma\left(x_{0}, \theta, d(\cdot)\right), \Xi_{D}\left(d_{\max }\right)\right)$ defined by (1), (4). We have shown in Theorem 2.1 that the choice of $\Omega_{0}^{*}=\left[-\eta_{0}^{*}, \eta_{0}^{*}\right]$, where $\eta_{0}^{*}:=\varrho\left(d_{\max }\right)=$ $d_{\max } / a$, suggested by Lyapunov theory, suffices to establish D1-D3. However, it is well known that the Lyapunov method only provides a sufficient condition for stability and in fact there are systems for which $x(t) \rightarrow \Omega_{0}=[-c, c]$ where $c<\eta_{0}^{*}$. However, in the following, we will illustrate that this is not true if the controllers generalized for tracking problems. Consider system (1) and define $e(t):=x(t)-x_{\text {ref }}(t)$ where $x_{\text {ref }}(\cdot)$ is a reference signal. The objective is for $x(\cdot)$ to approximately track the reference signal $x_{\text {ref }}(\cdot)$, i.e. $e(t) \rightarrow \Omega_{0}$ as $t \rightarrow \infty$. Let us define the following tracking controller:

$$
\begin{gathered}
u(t)=-a e(t)-\hat{\theta}(t) \phi(x(t))+\dot{x}_{\mathrm{ref}}(t) \\
\dot{\hat{\theta}}(t)=D_{\Omega_{0}}(e) e(t) \phi(x(t)) \quad \hat{\theta}(0)=0
\end{gathered}
$$

Observe that given $x_{\text {ref }}=0$, the tracking controller is identical to the dead-zone controller (4). In the presence of bounded disturbances, a routine calculation yields to

$$
\dot{e}(t)=-a e(t)+(\theta-\hat{\theta}(t)) \phi(x(t))+d(t)
$$

The choice of $\eta_{0}:=d_{\max } / a$ is suggested by Lyapunov analysis and implies $e(t) \rightarrow \Omega_{0}$ as $t \rightarrow \infty$. However, inspired by the above explanation one may choose $\eta_{0}:=c$. The following example illustrates the closed loop response to such a choice.

\section{Example 4.1}

Consider the closed loop interconnection system defined by (1), (57), and (58), where

$$
\phi(x(t))=x(t), \quad a=1, \quad \theta=2, \quad d(\cdot)=100, \quad c=10, \quad y_{\text {ref }}=10 \sin (t)
$$

The behaviour of the closed loop signals have been shown in Figure 2.

As illustrated in Figure 2, the tuning function $\hat{\theta}(\cdot)$ drifts. Comparing this situation to that of unmodified controller (3), one can easily build a similar setup as Proposition 3.3 to achieve $\mathscr{P}=\infty$. Therefore, this provides a motivation for the choice of dead-zone $\varrho\left(d_{\max }\right)=d_{\max } / a$.

\section{GENERALIZATIONS}

In this section, we outline the extension of the main results given in Section 2.3 to wider classes of systems, namely to non-linear systems in the form of integrator chain and to linear systems of relative degree one which are minimum phase and have positive high frequency gain. The proofs of these more general results follow the same structure to the above proof for the scalar case, but the details are substantively more involved. The proofs can be found in Reference [8], see also Reference [9]. 

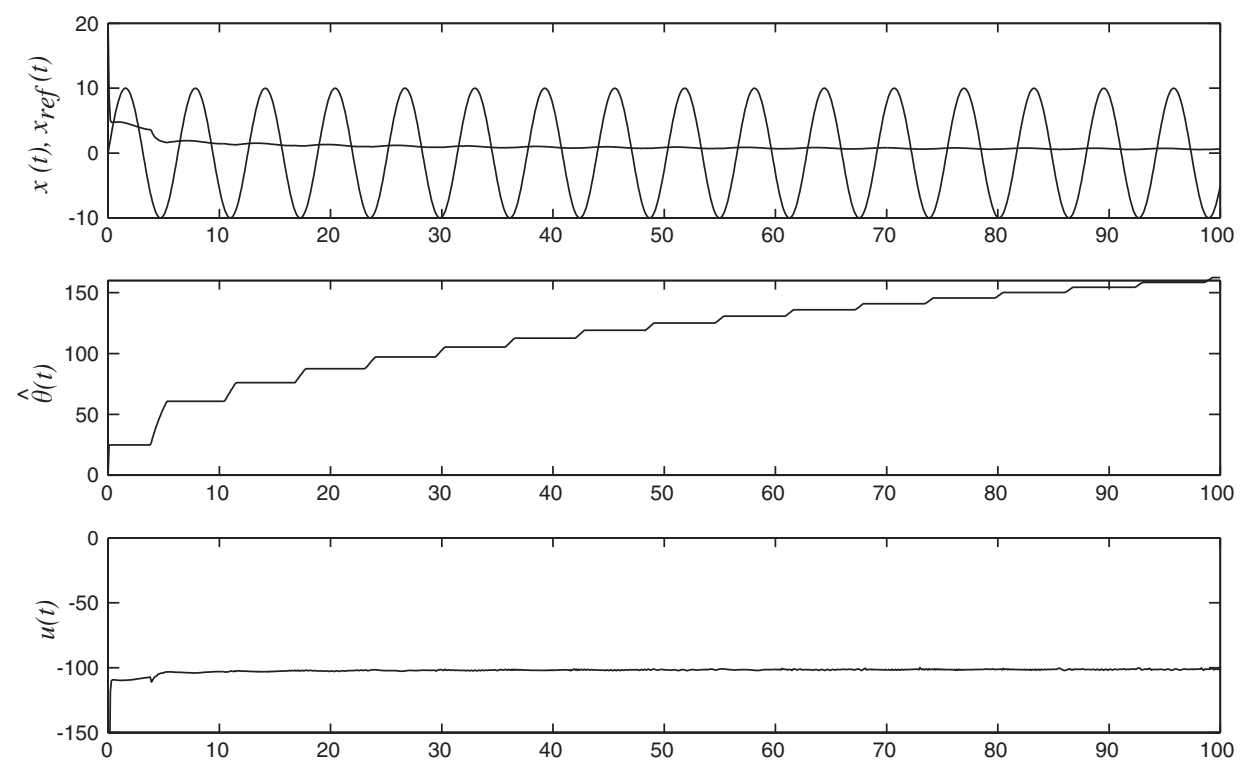

Figure 2. Tracking for Example 4.1.

\subsection{Integrator chain}

By an 'integrator chain', we mean the following SISO non-linear system:

$$
\begin{aligned}
\Sigma\left(x_{0}, \theta, d(\cdot)\right): \dot{x}_{i}(t) & =x_{i+1}(t), \quad 1 \leqslant i \leqslant n-1 \\
\dot{x}_{n}(t) & =\theta \phi(x(t))+u(t)+d(t) \\
y(t) & =x_{1}(t)
\end{aligned}
$$

Define the feedback law

$$
u(t):=-a^{\mathrm{T}} x(t)-\hat{\theta}(t) \phi(x(t))
$$

where $a=\left[a_{1}, \ldots, a_{n}\right]^{\mathrm{T}}$ is chosen such that the matrix

$$
A=\left[\begin{array}{ccccc}
0 & 1 & 0 & \ldots & 0 \\
0 & 0 & 1 & 0 & 0 \\
\vdots & \vdots & \vdots & \ddots & \vdots \\
0 & 0 & 0 & \ldots & 1 \\
-a_{1} & -a_{2} & -a_{3} & \ldots & -a_{n}
\end{array}\right]
$$

is Hurwitz. Let $P, Q$ be symmetric positive definite matrices satisfying the Lyapunov equation $A^{\mathrm{T}} P+P A=-Q$ and define the weighting vector $b:=\left(P+P^{\mathrm{T}}\right) B$, where $B:=(0, \ldots, 0,1)^{\mathrm{T}}$. The signal $\hat{\theta}: \mathbb{R}^{+} \rightarrow \mathbb{R}$ in (62) represents the adaptive estimator of $\theta$ and is updated by the online adaptive law:

$$
\dot{\hat{\theta}}(t)=\alpha x(t)^{\mathrm{T}} b \phi(x(t)), \quad \hat{\theta}(0)=0
$$


It can be shown that the controller $\Xi$, consisting of the feedback law (62) and the adaptive law (64), stabilizes systems $\Sigma\left(x_{0}, \theta, d(\cdot)\right)$ of form (61) when $\mathscr{D}=\{0\}$.

However, in the presence of bounded disturbances, we need to modify adaptive law (64). Based on the description of the dead-zone modification described in Section 2.2.1, we define the dead-zone region $\Omega_{0}\left(d_{\max }\right)$ :

$$
\Omega_{0}\left(d_{\max }\right)=\left\{x \mid x^{\mathrm{T}} P x \leqslant \eta_{0}^{2}\right\}
$$

where

$$
\eta_{0}:=\varrho\left(d_{\max }\right)=\frac{\sqrt{\bar{\lambda}(P)}}{\underline{\lambda}(Q)}|b| d_{\max }
$$

Consequently, the dead-zone controller is defined as follows:

$$
\begin{aligned}
\Xi_{D}\left(d_{\max }\right): & u(t)=-a^{\mathrm{T}} x(t)-\hat{\theta}(t) \phi(x(t)) \\
\dot{\hat{\theta}}(t) & =D_{\Omega_{0}\left(d_{\max }\right)}(x) \alpha x(t)^{\mathrm{T}} b \phi(x(t)), \quad \hat{\theta}(0)=0
\end{aligned}
$$

The projection operator defined in Section 2.2.2 can be used for integrator chain system (61) since $\theta \in \mathbb{R}$. A slight modification of (10) yields

$$
\begin{aligned}
\Xi_{P}\left(\theta_{\max }\right): & u(t)=-a^{\mathrm{T}} x(t)-\hat{\theta}(t) \phi(x(t)) \\
\dot{\hat{\theta}}(t) & =\operatorname{Proj}_{\Pi\left(\theta_{\max }\right)}\left(\alpha x(t)^{\mathrm{T}} b \phi(x(t))\right), \quad \hat{\theta}(0)=0
\end{aligned}
$$

5.1.1. Performance comparison. In order to generalize Theorems I and II, to non-linear systems of form (61), we need to establish parameter drift. To this end, we add an extra assumption to (2), and consider the following conditions on the function $\phi(\cdot): \mathbb{R}^{n} \rightarrow \mathbb{R}$ :

$$
\begin{aligned}
& \text { (a) } x=0 \Leftrightarrow \phi(x)=0 \\
& \text { (b) } x^{\mathrm{T}} b \phi(x) \geqslant 0 \\
& \text { (c) }\left.\frac{\partial \phi(x)}{\partial x_{n}}\right|_{x=0}>0 \\
& \text { (d) } \inf _{x \in \mathbb{R}^{n}} \frac{|\phi(x)|}{|x|} \geqslant \beta>0
\end{aligned}
$$

These conditions specify that the uncontrolled system $(u=0)$ has an unstable equilibrium at the origin, and that the non-linearity has at least linear growth, and that a certain positivity condition holds. Of these four conditions, (b) is the most restrictive. The following theorem shows the extension of our main results to non-linear systems in the form of an integrator chain (61).

\section{Theorem 5.1}

Consider the system $\Sigma\left(x_{0}, \theta, d(\cdot)\right)$ and the controllers $\Xi_{D}\left(d_{\max }\right)$ and $\Xi_{P}\left(\theta_{\max }\right)$ defined by $(61),(67)$ and (68) respectively. Consider the transient performance cost functional (14). Then 
1. If $\phi(\cdot)$ satisfies conditions 69 -a,b,c, then $\forall d_{\max } \geqslant \varepsilon, \exists \theta_{\max }^{*} \geqslant \delta$ s.t. $\forall \theta_{\max } \geqslant \theta_{\max }^{*}$,

$$
\mathscr{P}\left(\Sigma\left(\mathscr{X}_{0}(\gamma), \Delta(\delta), \mathscr{D}(\varepsilon)\right), \Xi_{P}\left(\theta_{\max }\right)\right)>\mathscr{P}\left(\Sigma\left(\mathscr{X}_{0}(\gamma), \Delta(\delta), \mathscr{D}(\varepsilon)\right), \Xi_{D}\left(d_{\max }\right)\right)
$$

2. If $\phi(\cdot)$ satisfies conditions 69 -d, then $\exists \delta>0$ s.t. $\forall \theta_{\max } \geqslant \delta \exists d_{\max }^{*} \geqslant \varepsilon$ such that $\forall d_{\max } \geqslant d_{\max }^{*}$,

$$
\mathscr{P}\left(\Sigma\left(\mathscr{X}_{0}(\gamma), \Delta(\delta), \mathscr{D}(\varepsilon)\right), \Xi_{D}\left(d_{\max }\right)\right)>\mathscr{P}\left(\Sigma\left(\mathscr{X}_{0}(\gamma), \Delta(\delta), \mathscr{D}(\varepsilon)\right), \Xi_{P}\left(\theta_{\max }\right)\right)
$$

\section{Proof}

See Reference [8].

\subsection{Linear systems}

Suppose $\Sigma$ is a SISO linear time invariant plant described by

$$
y=\frac{b_{m} s^{m}+b_{m-1} s^{m-1}+\cdots+b_{0}}{s^{n}+a_{n-1} s^{n-1}+\cdots+a_{0}}(u+d)
$$

where $a_{i}, b_{j}, 0 \leqslant i \leqslant n-1,0 \leqslant j \leqslant m$, are unknown constants and $d(\cdot)$ belongs to a class of bounded disturbances $\mathscr{D} \subset \mathscr{L}^{\infty}[0, \infty)$. We assume that only output $y(\cdot)$ is available for measurement. A minimal state space realisation of the plant in canonical observer form can be obtained as follows:

$$
\begin{aligned}
\Sigma\left(x_{0}, \theta, d(\cdot)\right): \dot{x}(t) & =A x(t)+B(u(t)+d(t)), \quad x(0)=x_{0} \\
y(t) & =C x(t)
\end{aligned}
$$

in which $x(t), B, C^{\mathrm{T}} \in \mathbb{R}^{n}, A \in \mathbb{R}^{n \times n}$, and

$$
A=\left[\begin{array}{ccccc}
-a_{n-1} & 1 & 0 & \ldots & 0 \\
-a_{n-2} & 0 & 1 & 0 & 0 \\
\vdots & \vdots & \vdots & \ddots & \vdots \\
-a_{1} & 0 & 0 & \ldots & 1 \\
-a_{0} & 0 & 0 & \ldots & 0
\end{array}\right], \quad B=\left[\begin{array}{c}
0(\rho-1) \\
b_{m} \\
\vdots \\
b_{1} \\
b_{0}
\end{array}\right], \quad C=\left[\begin{array}{llll}
1 & 0 & \ldots & 0
\end{array}\right]
$$

where $\rho=n-m$ is the relative degree of the system and

$$
\theta=\left(a_{0}, \ldots, a_{n-1}, b_{0}, \ldots, b_{m}\right)
$$

represents the uncertain system parameters. Consider the following assumptions:

C1. The plant is minimum phase, i.e. $b_{m} s^{m}+b_{m-1} s^{m-1}+\cdots+b_{0}$ is Hurwitz.

C2. The plant order $n$ is known; the plant is of relative degree one (i.e. $\rho=1$ ), and the highfrequency gain is positive (i.e. $b_{m}=b_{n-1}>0$ ).

It was shown [13] that disturbance free $(\mathscr{D}=\{0\})$ systems of form $(71)$, i.e. $\Sigma\left(x_{0}, \theta, d(\cdot)\right)$ which satisfy $\mathrm{C} 1, \mathrm{C} 2$, are stabilized by the following simple adaptive high-gain controller:

$$
\begin{aligned}
\Xi: u(t) & =-\hat{\delta}(t) y(t) \\
\dot{\hat{\delta}}(t) & =y(t)^{2} \quad \hat{\delta}(0)=0
\end{aligned}
$$


The above controller is a basis for 'non-identifier-based' adaptive controllers and $\hat{\delta}(\cdot)$ is called 'tuning function'. Special features of such direct adaptive controllers are their simplicity and the absence of any plant identification mechanism. For an early survey see Reference [14].

In the presence of bounded disturbances, the dead-zone modification can be defined in the standard form as described in Section 2.2.1. However, as an alternative- to avoid discontinuous switching, we use so-called 'smooth dead-zone' defined by

$$
D_{\Omega_{0}\left(d_{\max }\right)}^{\prime}(y)= \begin{cases}0, & y \in \Omega_{0}\left(d_{\max }\right) \\ |y|-\eta_{0}, & y \notin \Omega_{0}\left(d_{\max }\right)\end{cases}
$$

leading to the modified adaptive law of form [14]

$$
\begin{aligned}
\Xi_{D^{\prime}}\left(d_{\max }\right): & u(t)=-\hat{\delta}(t) y(t) \\
\dot{\hat{\delta}}(t) & =D_{\Omega_{0}\left(d_{\max }\right)}^{\prime}(y)|y(t)|, \quad \hat{\delta}(0)=0, \quad \eta_{0}=d_{\max }
\end{aligned}
$$

The definition of projection modification in 'non-identifier-based' case is as follows: define

$$
\delta_{\theta}=\inf \{\delta \geqslant 0 \mid A-\tilde{\delta} B C \text { is Hurwitz } \forall \tilde{\delta} \geqslant \delta\}
$$

and let $\delta_{\max }$ be a strict upper bound for $\delta_{\theta}$. Define the set $\Pi\left(\delta_{\max }\right):=\left[0, \delta_{\max }\right]$ and let $T_{m}$ be the first time instance that $\hat{\delta}$ hits the boundary $\delta_{\max }$. The projection controller is defined by

$$
\begin{aligned}
\Xi_{P}\left(\delta_{\max }\right): & u(t)=-\hat{\delta}(t) y(t) \\
\dot{\hat{\delta}}(t) & =y(t)^{2}, \quad \hat{\delta}(0)=0 \quad \forall t \in\left[0, T_{m}\right] \\
\hat{\delta}(t) & =\delta_{\max } \quad \forall t \in\left[T_{m}, \infty\right)
\end{aligned}
$$

5.2.1. Performance comparison. Consider the following cost functional:

$$
\mathscr{P}\left(\Sigma\left(\mathscr{X}_{0}(\gamma), \Lambda, \mathscr{D}(\varepsilon)\right), \Xi\right)=\sup _{x_{0} \in \mathscr{X}_{0}(\gamma)} \sup _{\theta \in \Lambda} \sup _{d \in \mathscr{D}(\varepsilon)}\left(\|x(\cdot)\|_{\mathscr{L}^{\infty}}+\|u(\cdot)\|_{\mathscr{L}^{\infty}}+\|\dot{u}(\cdot)\|_{\mathscr{L}^{\infty}}\right)
$$

where

$$
\begin{aligned}
\mathscr{X}_{0}(\gamma) & :=\left\{x_{0} \mid\left\|x_{0}\right\| \leqslant \gamma\right\}, \quad \gamma>0 \\
\mathscr{D}(\varepsilon) & :=\left\{d(\cdot) \mid\|d(\cdot)\|_{\mathscr{L}^{\infty}} \leqslant \varepsilon\right\}, \quad \varepsilon \geqslant 0
\end{aligned}
$$

and $\Lambda$ is any compact subset of $\Delta(\delta)$, where

$$
\Delta(\delta):=\left\{\theta \in \mathbb{R}^{2 n} \mid A-\delta B C \text { is Hurwitz and } \mathrm{C} 1 \text { and } \mathrm{C} 2 \text { hold }\right\}, \quad \delta \geqslant 0
$$

and $\theta$ is given by (73). Note that there are elements on the boundary of $\Delta(\delta)$ which do not satisfy $\mathrm{C} 1$ and $\mathrm{C} 2$ and for which the closed loop is not stable, hence generating an infinite cost. Therefore the second supremum cannot be taken over $\Delta(\delta)$ instead we take the supremum over a compact subset $\Lambda$ of $\Delta(\delta)$, which necessarily does not contain any elements on the boundary of $\Delta(\delta)$ which violate $\mathrm{C} 1$ and $\mathrm{C} 2$.

The following theorem shows the generalization of the main results to minimum phase linear systems of relative degree one with known high-frequency gain: 
Theorem 5.2

Consider the system $\Sigma\left(x_{0}, \theta, d(\cdot)\right)$ and the controllers $\Xi_{D}\left(d_{\max }\right)$ and $\Xi_{P}\left(\theta_{\max }\right)$ defined by (71), (76) and (78) respectively, where $\mathrm{C} 1$ and $\mathrm{C} 2$ hold. Let $\Lambda \subset \Delta(\delta)$ be compact. Consider the transient performance cost functional (79). Then,

1. for all $d_{\max } \geqslant \varepsilon$, there exists $\delta_{\max }^{*} \geqslant \delta$ such that for all $\delta_{\max } \geqslant \delta_{\max }^{*}$,

$$
\mathscr{P}\left(\Sigma\left(\mathscr{X}_{0}(\gamma), \Lambda, \mathscr{D}(\varepsilon)\right), \Xi_{P}\left(\delta_{\max }\right)\right)>\mathscr{P}\left(\Sigma\left(\mathscr{X}_{0}(\gamma), \Lambda, \mathscr{D}(\varepsilon)\right), \Xi_{D^{\prime}}\left(d_{\max }\right)\right)
$$

2. there exists $\delta>0$ such that for all $\delta_{\max } \geqslant \delta$, there exists $d_{\max }^{*} \geqslant \varepsilon$ so that for all $d_{\max } \geqslant d_{\max }^{*}$,

$$
\mathscr{P}\left(\Sigma\left(\mathscr{X}_{0}(\gamma), \Lambda, \mathscr{D}(\varepsilon)\right), \Xi_{D^{\prime}}\left(d_{\max }\right)\right)>\mathscr{P}\left(\Sigma\left(\mathscr{X}_{0}(\gamma), \Lambda, \mathscr{D}(\varepsilon)\right), \Xi_{P}\left(\delta_{\max }\right)\right)
$$

Proof

See either Reference [8] or [9].

\section{CONCLUDING REMARKS}

By considering a non-singular performance cost functional for a simple class of scalar nonlinear systems, we have established two rigourous results comparing the performance of the dead-zone and the projection based robust adaptive control systems:

- The dead-zone based controller outperforms the projection based controller when the $a$ priori information on the uncertainty level is sufficiently conservative.

- The projection based controller outperforms the dead-zone based controller when the $a$ priori information on the disturbance level is sufficiently conservative.

Extensions of these results to more physically meaningful classes of systems (e.g. minimum phase linear systems with relative degree one and of positive high-frequency gain, and non-linear systems in the form of integrator chain) were outlined in Section 5.

This case study has shown that a quantitative cost based approach is a theoretically tractable approach to assess relative benefits of different robust adaptive controllers. A related result can be found in Reference [15] where a robust backstepping design is compared to an adaptive backstepping design w.r.t. to a non-singular transient performance cost functional. It should be observed that the comparison of the transient performance cannot be sensibly posed without employing a non-singular cost to formulate the problem, and there are only a few other published results on non-singular performance bounds for adaptive controllers in the References [11, 16, 17].

The emphasis in this paper has been on comparisons between controllers when applied to a simple plant. This enabled the mechanism underlying the trade-offs to be elucidated in a clear manner. It should be observed that many adaptive control designs (e.g. backstepping designs) with the same robust modifications reduce to the controllers considered here when applied to the scalar plant. Therefore, we have shown that these trade-offs between designs are present for a wide class of common adaptive control approaches. We anticipate that these trade-offs persist in much more general settings, in particular 
there are a number of immediate directions in which the results can be fruitfully generalized, for example:

- Relaxation of assumption 69-b which is required in the current comparative proofs for the integrator chain. Currently, the proof of parameter drift relies on the assumption $x^{\mathrm{T}} b \phi(x) \geqslant 0$ which restricts the choices of $\phi(\cdot)$.

- Generalization of the result to strict feedback systems, for example for backstepping controllers.

- Relaxation of the requirement of a matched disturbance in the non-scalar state cases.

- Establishing whether the same results can be given for the alternative costs, for example, $\mathscr{P}=\|x(\cdot)\|_{\mathscr{L}^{\infty}}+\|u(\cdot)\|_{\mathscr{L}^{\infty}}$.

- Extension of the techniques developed for the comparison to other robust adaptive algorithms, e.g. $\sigma$-modification, relative dead-zone, etc.

The aim is to establish good characterizations of the classes of problem in which one controller should be used in preference to another. By providing a framework to address these questions, the results of this paper represents a step towards these more general results.

\section{REFERENCES}

1. Egardt B. Stability of Adaptive Controllers. Lecture Notes in Control and Information Sciences. Springer: New York, 1979.

2. Ioannou PA, Kokotović PV. Instability analysis and improvement of robustness of adaptive control. Automatica 1984; 20:583-594.

3. Ioannou PA, Sun J. Robust Adaptive Control. Prentice-Hall: Englewood Cliffs, NJ, 1996.

4. Kreisselmeier G, Narendra KS. Stable model reference adaptive control in the presence of bounded disturbances. IEEE Transactions on Automatic Control 1982; 27(6):1169-1175.

5. Narendra KS, Annaswamy AM. Stable Adaptive Systems. Prentice-Hall, Englewood Cliffs, NJ, 1989.

6. Peterson BB, Narendra KS. Bounded error adaptive control. IEEE Transactions on Automatic Control 1982; 27(6):1161-1168.

7. Mareels IMY, Van Gils S, Polderman JW, Ilchmann A. Asymptotic dynamics in adaptive gain control. In Advances in Control, Frank PM (ed). Springer: Berlin, 1999; 29-63.

8. Sanei A. Towards a Performance Theory of Robust Adaptive Control. Ph.D. Thesis, Department of Electronics and Computer Science, University of Southampton, UK, 2003. Available from www.ecs.soton.ac.uk $/ \sim \mathrm{mcf}$.

9. Sanei A, French M. A performance comparison of robust adaptive controllers: linear systems. Mathematics of Control, Signals, and Systems, 2003, submitted for publication.

10. Filippov AF. Differential Equations with Discontinuous Righthand Sides. Kluwer: Dordrecht, 1988.

11. French M, Rogers E, Szepesvári Cs. Uncertainty, performance, and model dependency in approximate adaptive nonlinear control. IEEE Transactions on Automatic Control 2000; 45(2):353-358.

12. Krstić M, Kanellakopoulos I, Kokotović P. Nonlinear and Adaptive Control Design. Wiley: New York, 1995.

13. Byrnes CI, Willems JC. Adaptive stabilization of multivariable linear systems. In Proceedings of the 23rd IEEE Conference on Decision and Control, Las Vegas, Nevada, 1984; 1574-1577.

14. Ilchmann A. Non-Identifier-Based High-Gain Adaptive Control. Lecture Notes in Control and Information Sciences. Springer: London, 1993.

15. French M. An analytical comparison between the nonsingular quadratic performance of robust and adaptive backstepping designs. IEEE Transactions on Automatic Control 2002; 47(4):670-675.

16. Didinsky G, Basar T. Minimax adaptive control of uncertain plants. In Proceedings of the 33rd IEEE Conference on Decision and Control, Lake Buena Vista, Florida, 1994; 2839-2844.

17. Li ZH, Krstić M. Optimal design of adaptive tracking controllers for non-linear systems. Automatica 1997; 33(8):1459-1473. 